\title{
Using High Resolution Cardiac CT Data to Model and Visualize Patient-Specific Interactions between Trabeculae and Blood Flow
}

\author{
Scott Kulp ${ }^{1}$, Mingchen $\mathrm{Gao}^{1}$, Shaoting Zhang ${ }^{1}$, \\ Zhen Qian ${ }^{2}$, Szilard Voros ${ }^{2}$, Dimitris Metaxas ${ }^{1}$, and Leon Axel ${ }^{3}$ \\ 1 CBIM Center, Rutgers University, Piscataway, NJ, 08550, USA \\ 2 Piedmont Heart Institute, Atlanta, GA, 30309, USA \\ 3 New York University, 660 First Avenue, New York, NY, 10016, USA
}

\begin{abstract}
In this paper, we present a method to simulate and visualize blood flow through the human heart, using the reconstructed 4D motion of the endocardial surface of the left ventricle as boundary conditions. The reconstruction captures the motion of the full 3D surfaces of the complex features, such as the papillary muscles and the ventricular trabeculae. We use visualizations of the flow field to view the interactions between the blood and the trabeculae in far more detail than has been achieved previously, which promises to give a better understanding of cardiac flow. Finally, we use our simulation results to compare the blood flow within one healthy heart and two diseased hearts.
\end{abstract}

\section{Introduction}

Following a heart attack or the development of some cardiovascular diseases, the movement of the heart walls during the cardiac cycle may change. This affects the motion of blood through the heart, potentially leading to an increased risk of thrombus. While Doppler ultrasound and MRI can be used to monitor valvular blood flow, the image resolutions are low and they cannot capture the interactions between the highly complex heart wall and the blood flow. For this reason, with the rapid development of high-resolution cardiac CT, patientspecific blood flow simulation can provide a useful tool for the study of cardiac blood flow.

Recently, Mihalef et al. 9] used smoothed 4D CT data to simulate left ventricular blood flow, and compared the flow through the aortic valve in a healthy heart and two diseased hearts. However, the models derived from CT data in [9] were too highly smoothed to capture the local structural details and were not useful for understanding the true interactions between the blood flow and the walls.

Later, in [7, more accurate heart models were achieved by generating a mesh from high-resolution CT data at mid-diastole. Then, motion was transferred to this model from the smooth mesh motion obtained from the same CT data to create the animation. This allowed for more realistic features to be present on the

G. Fichtinger, A. Martel, and T. Peters (Eds.): MICCAI 2011, Part I, LNCS 6891, pp. 468 475, 2011. (C) Springer-Verlag Berlin Heidelberg 2011 


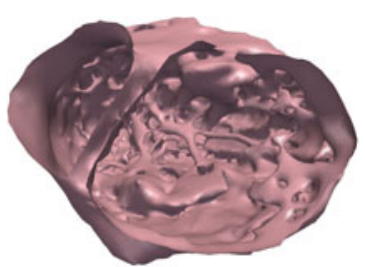

(a)

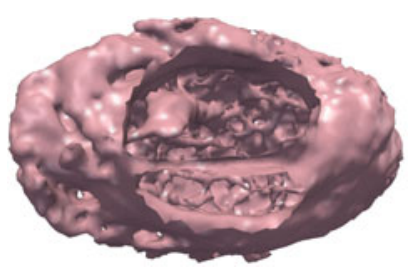

(b)

Fig. 1. Meshes reconstructed from CT data (valves removed). (a) Healthy heart (b) Diseased heart.

heart walls in the simulation, including the papillary muscles and some trabeculae. However, while this approach was an improvement from the smooth-wall assumption, the trabeculae were missing details and did not move accurately.

Earlier work in blood flow simulation used less refined models. For example, [5] was the first to extract boundaries from MRI data to perform patient-specific blood flow simulations. Later, 8 8 used simple models of the left side of the heart, with smooth ventricular walls, and imposed boundary conditions in the valve regions. In 2010, 6 developed a technique to drive the deformation of a smoothed left ventricle by implicitly coupled fluid motion.

In this paper, we use an improved method of creating the mesh to capture these smaller details and generate a more accurate simulation. To the best of our knowledge, we are able to visualize blood flow in unprecedented detail.

\section{Data Acquisition}

The CT images were acquired on a 320-MSCT scanner (Toshiba Aquilion ONE, Toshiba Medical Systems Corporation) using contrast agent. This advanced diagnostic imaging system is a dynamic volume CT scanner that captures a wholeheart scan in a single rotation, and achieves an isotropic $0.3 \mathrm{~mm}$ volumetric resolution. A conventional contrast-enhanced CT angiography protocol was adapted to acquire the $\mathrm{CT}$ data in this work. After the intravenous injection of contrast agent, the $3 \mathrm{D}+$ time $\mathrm{CT}$ data were acquired in a single heart beat cycle when the contrast agent was circulated to the left ventricle and aorta. After acquisition, $3 \mathrm{D}$ images were reconstructed at 10 time phases in between the $\mathrm{R}$-to- $\mathrm{R}$ waves using ECG gating. The acquired isotropic data had an in-plane dimension of 512 by 512 pixels.

The detailed cardiac shape features can be used as the boundary conditions and incorporated in a fluid simulator to derive the hemodynamics throughout the whole heart cycle. Our goal in defining these boundary conditions is to capture the fine detail structures of the myocardium, as well as the one-to-one vertex correspondence between frames, which is required in the fluid simulation. There has been much recent work in cardiac reconstruction, such as [11, who combined high-resolution MRI images with serial histological sectioning data to build histo-anatomically detailed individualized cardiac models to investigate 
cardiac function. In this work, we use the techniques described in [3]. Here, snake based semi-automatic segmentation is used to acquire the initial segmentation from high resolution CT data for an initial (3D) frame of data. The initial mesh is generated as an isosurface of the segmentation, which is deformed to match the shape of the heart in each consecutive frame. also during the deformation, we achieve the necessary one-to-one correspondence between frames.

The aortic and mitral valves are thin and move fast, and so the CT data is not currently able to adequately capture these details. We add 3D models of the valves created from ultrasound data to each mesh in the sequence, and open and close the valves at the appropriate time steps.

Reconstruction results for a healthy and a diseased heart can be seen in Figure 1. Note the high level of structural detail at the apex. To the best of our knowledge, this has never been simulated before.

\section{Fluid Simulation}

The motion of an incompressible fluid is governed by the laws of conservation of momentum and mass, modeled by the Navier-Stokes (NS) equations:

$$
\begin{gathered}
\rho\left(\frac{\partial u}{\partial t}+u \cdot \nabla u\right)=-\nabla P+\mu \nabla^{2} u, \\
\nabla \cdot u=0 .
\end{gathered}
$$

Here, $\rho$ is the fluid density, $u$ is the $3 \mathrm{D}$ velocity vector field, $P$ is the pressure field, and $\mu$ is the coefficient of viscosity. We seek to solve these equations for velocity and pressure.

Foster and Metaxas [2] were the first to develop a fast method of solving the NS equations for graphics applications by applying a staggered grid across the domain and explicitly solving for velocity at the cell faces. They then used SOR to solve for pressure and correct the velocities to maintain incompressibility.

Our fluid-solid interaction system uses a "boundary immersed in a Cartesian grid formulation", allowing for an easy treatment of complex moving geometries embedded in the computational domain. Recent work that employs such a formulation is [13], which applies the formulation of [12] to both graphics and medical simulations. Recently, [1] implemented the approach of [4] to obtain a system that can efficiently deal with complex geometric data, such as a system of blood vessels.

The heart models used here are embedded in a computational mesh of $100^{3}$ cells on which the full NS equations are solved using FDM. The blood is modeled as a Newtonian fluid, with viscosity of $4 \mathrm{mPa} \cdot \mathrm{s}$ and density of $1050 \mathrm{~kg} / \mathrm{m}^{3}$, which are physiologically accepted values for normal human blood $[9$. The heart model is given to the solver as a set of meshes with point correspondences, which allows for easy interpolation and also obtaining the velocity of the heart mesh at every point in time. Our system represents the 3D meshes as a Marker Level Set(MLS) [10], where markers are placed on the boundary and are used to correct the level set at every time step. Since markers are only placed on the surface, MLS has been proven to be more efficient and more accurate for complex boundaries. 


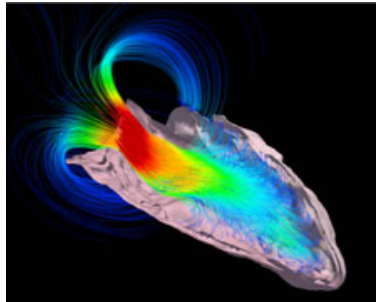

(a)

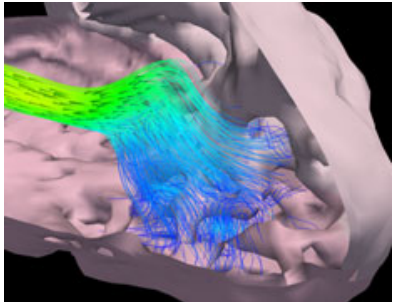

(b)

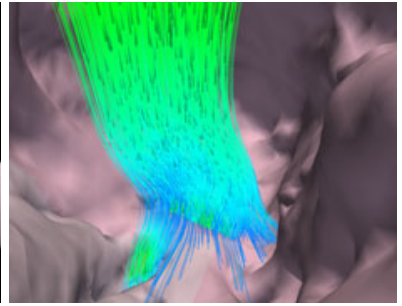

(c)

Fig. 2. Visualization of streamlines within the healthy heart. (a) Streamlines of cardiac blood flow during diastole. (b) Blood flow near apex during diastole. (c) Blood flow during systole at the apex, against the trabeculae.

The MLS and its velocity are rasterized onto the Eulerian grid and are used to impose the appropriate boundary conditions in the fluid solver. A simulation of two complete cardiac cycles takes about four days to complete on a machine with a Core 2 Quad processor and $8 \mathrm{~GB}$ of RAM.

\section{Visualizations}

With the fluid velocity fields and level sets generated for each time step, we use Paraview to visualize the simulations. We analyzed a healthy heart and two diseased hearts, and we describe below our visualization methods and our results.

Blood Flow Velocity. We performed a visualization of the velocity field within the heart, as seen in Figure 3. The velocity of the blood at a given point is represented by a cone pointed in the direction of the flow. The size of cone increases linearly as the velocity increases. We also adjust the color of a cone by first setting its hue to 160 (blue), and then linearly lowering this value to a minimum of 0 (red) as velocity increases. The magnitude of fluid velocity ranges from $0-.9 \mathrm{~m} / \mathrm{s}$.

Streamline visualizations are shown in Figure 2. The color at a point within a streamline is chosen in the same way as the cones described above. In order to disambiguate direction, we add cones that point in the direction of flow

\subsection{Blood Residence Time}

In addition to the blood flow velocities, we wish to visualize the residence time of blood within the heart. By doing so, we can quantitatively determine regions of the heart that are at greater risk of thrombus, as slower flows are known to be a significant factor predisposing to thrombus formation.

At the initial time step, ten thousand particles are generated randomly within the heart. At the beginning of each time step, new particles are generated at the valves, allowing fresh blood particles to enter the heart during diastole. Each new particle has an initial age of zero, and this age is incremented at every time step. 
At each consecutive time step, we determine a particle's velocity by interpolation, given the fluid velocities at the center of each cell. Each particle's new position is calculated using Euler time integration. Then, any particle in a cell exterior to the heart is removed from the system, and the average particle residence time within each cell can then be easily determined. We run this for four cardiac cycles and create volumetric visualizations, as seen in Figure 4 . Here, blue represent regions in which average residency is less than 1 cardiac cycle, green-yellow represents 1-3 cardiac cycles, and red represents 3-4 cycles.

We can also take advantage of these particles in validation of our simulation, by computing an estimated ejection fraction. During systole, we know exactly how many particles there originally existed in the system, and how many are being expelled at each time step. To estimate the ejection fraction, we simply divide the total number of deleted particles by the original number of particles.

\section{Discussion}

The streamline visualizations provide detailed information on the trabeculaeblood interaction. Figure 2(b), taken during diastole, demonstrates how the complex surface causes the flow to move through and around the empty spaces between the trabeculae. Then, in Figure 2(c), during systole, we see another example of how the blood is forcefully expelled out of the spaces between the trabeculae, rather than simply flowing directly towards the aortic valve as older methods with simpler meshes have suggested.

The simulation and visualization methods are performed described above on three different hearts. The first is a healthy heart with no visible medical problems with an ejection fraction of about $50 \%$. The second is a heart that has simulated hypokinesis, where the motion of the heart walls is decreased at the apex by a maximum of $50 \%$. The third comes from a patient who has post tetralogy of Fallot repair. This heart is known to suffer from right ventricle hypertrophy, significant dyssynchrony in the basal-midseptum of the left ventricle, and a decreased left ventricle ejection fraction of about $30 \%$.

The streamline visualizations provide detailed information on the trabeculaeblood interaction. Figure2(b), taken during diastole, demonstrates how the complex surface causes the flow to fill the empty spaces between the trabeculae. Then, in Figure 2(c), during systole, we see another example of how the blood is expelled out of the spaces between the trabeculae, rather than simply flowing directly towards the aortic valve as older methods with simpler meshes have suggested.

Validation is a difficult task, as current imaging techniques, such as PC-MRI, are not able to capture flow information at the required level of detail for useful comparison. We performed a partial validation by comparing the estimated ejection fraction to the true ejection fraction. The computed ejection fraction is approximately $45 \%$ for the healthy heart, $40 \%$ for the hypokinesis heart, and $30 \%$ for the dyssynchronous heart. These values for the healthy and dyssynchronous heart are in agreement with the true values, so we have confidence in 


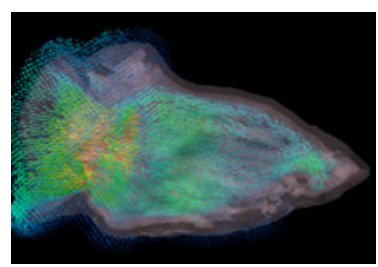

(a)

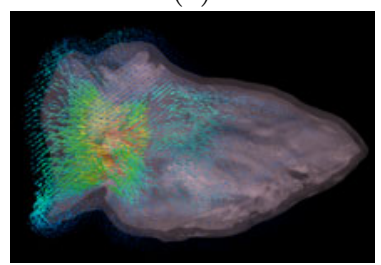

(d)

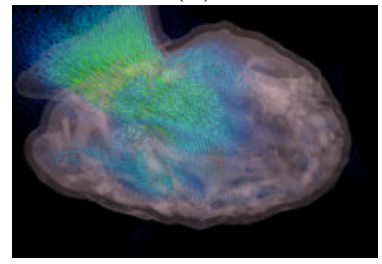

(g)

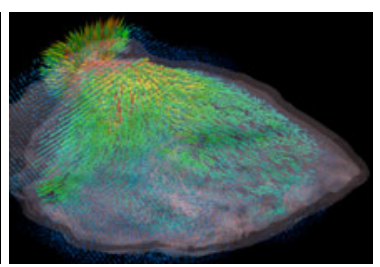

(b)

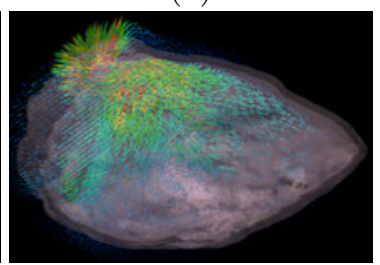

(e)

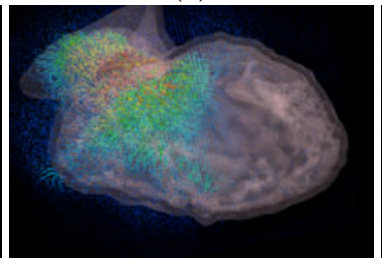

(h)

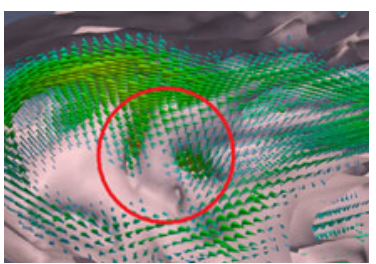

(c)

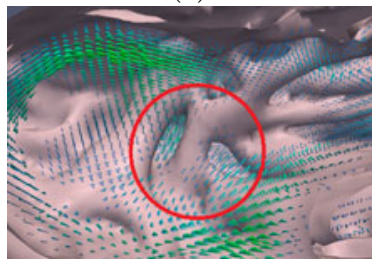

(f)

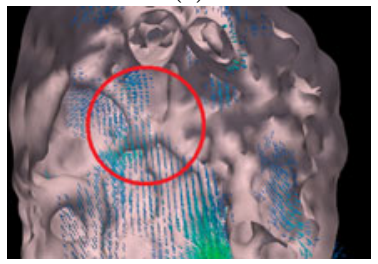

(i)

Fig. 3. Velocity fields at various time steps for three different hearts. Top row: Healthy Heart, Middle row: Hypokinetic heart, Bottom row: Dyssynchronous heart. Left column: Diastole, Middle column: Systole, Right column: Velocity field at trabeculae during systole.

the rest of our results. Performing similar validation techniques to a smoothed healthy heart model, we computed an ejection fraction of about $40 \%$, slightly lower than that of our complex model. However, it may not be especially useful to compare the accuracy of different modeling methods using this approach, as the ejection fraction does not give information about the flow local to the apex, the region of primary interest.

Velocity field visualizations are illustrated in Figure 3. We can see that in the healthy heart, the inflow during diastole is significant and fairly uniformly distributed, circulating blood throughout the heart. During systole, the velocity field throughout the heart remains high, and fluid in the apex moves toward the valves. In Figure 3(c), we see more detail of the interactions between blood flow and the trabeculae, as the blood is visibly expelled from these regions. However, in the heart suffering from hypokinesis, we find that the velocity field is much weaker toward the apex during both diastole and systole. In Figure 3(f), we also see that the trabeculae are no longer adequately expelling blood as they do in the healthy heart case. We also see in Figure 3(g)-(i) that the flow patterns in the heart with dyssynchronous heart wall movement appears non-normal, with overall lower velocities and even less fluid being pushed out from the trabeculae. 


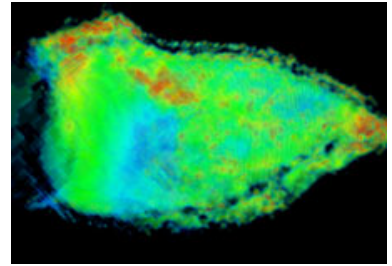

(a)

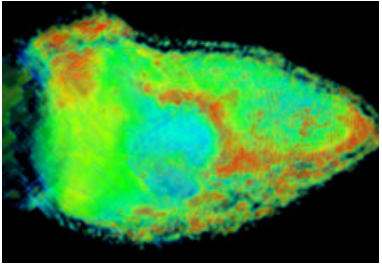

(b)

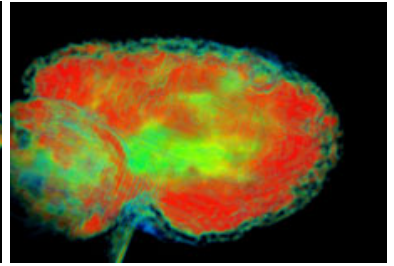

(c)

Fig. 4. Visualization of average particle residence time. Colors closer to red represent longer average residence time. (a) Healthy Heart (b) Heart with Hypokinesis (c) Heart with dyssynchronous wall movement.

We then compare the visualizations of the average particle residence times for each of the three simulations, as seen in Figure 4 Each of these images were made at the same time step, at the start of systole, after four cardiac cycles. We find that in Figure 4(a), in the healthy heart, nearly the entire domain contains blood with average residence time of less than three cycles, suggesting that the blood is not remaining stagnant, and turning over well between cardiac cycles. In contrast, Figure 4(b) shows that in the heart suffering from hypokinesis, the average residence time is significantly higher near the walls, particularly near the hypokinetic apex. Finally, in Figure 4(c), we find that a very significant region of the blood has a long residence time, suggesting that due to the low ejection fraction and relatively low fluid velocities, blood is not being adequately circulated and thus is remaining stagnant near the walls, again, particularly toward the apex of the heart.

\section{Conclusions}

In this paper, we have described our new framework to generate detailed mesh sequences from CT data, and used them to run patient-specific blood flow simulations. We then created several visualizations to reveal the interactions between the complex trabeculae of the heart wall and the blood, which has never been possible before, and used them to compare the flow fields between a healthy heart and two diseased hearts, which would potentially be extremely useful to doctors to help in diagnosis and treatment plans. This is the first time that intracardiac blood flow fields and their interaction with the heart wall have been investigated at this level of resolution.

Acknowledgments. This material is based upon work supported by the U.S. Department of Homeland Security under Grant Award Number 2007-ST-104000006. The views and conclusions contained in this document are those of the authors and should not be interpreted as necessarily representing the official policies, either expressed or implied, of the U.S. Department of Homeland Security. 


\section{References}

1. de Zelicourt, D., Ge, L., Wang, C., Sotiropoulos, F., Gilmanov, A., Yoganathan, A.: Flow simulations in arbitrarily complex cardiovascular anatomies - an unstructured cartesian grid approach. Computers \& Fluids 38(9), 1749-1762 (2009)

2. Foster, N., Metaxas, D.: Realistic animation of liquids. Graph. Models Image Process. 58, 471-483 (1996)

3. Gao, M., Huang, J., Zhang, S., Qian, Z., Voros, S., Metaxas, D., Axel, L.: 4D cardiac reconstruction using high resolution ct images. In: Metaxas, D., Axel, L. (eds.) FIMH (2011)

4. Gilmanov, A., Sotiropoulos, F.: A hybrid cartesian/immersed boundary method for simulating flows with 3D, geometrically complex, moving bodies. J. Comput. Phys. 207(2), 457-492 (2005)

5. Jones, T.N., Metaxas, D.: Patient-specific analysis of left ventricular blood flow. In: Wells, W.M., Colchester, A.C.F., Delp, S.L. (eds.) MICCAI 1998. LNCS, vol. 1496, pp. 156-166. Springer, Heidelberg (1998)

6. Krittian, S., Janoske, U., Oertel, H., Bhlke, T.: Partitioned fluid-solid coupling for cardiovascular blood flow. Annals of Biomedical Engineering 38, 1426-1441 (2010)

7. Kulp, S., Metaxas, D., Qian, Z., Voros, S., Axel, L., Mihalef, V.: Patient-specific modeling and visualization of blood flow through the heart. In: Wright, S., Pan, X., Liebling, M. (eds.) ISBI (2011)

8. Long, Q., Merrifield, R., Yang, G.Z., Xu, X.Y., Kilner, P.J., Firmin, D.N.: The influence of inflow boundary conditions on intra left ventricle flow predictions. Journal of Biomechanical Engineering 125(6), 922-927 (2003)

9. Mihalef, V., Ionasec, R., Wang, Y., Zheng, Y., Georgescu, B., Comaniciu, D.: Patient-specific modeling of left heart anatomy, dynamics and hemodynamics from high resolution 4d CT. In: Niessen, W., Meijering, E. (eds.) ISBI, pp. 504-507 (2010)

10. Mihalef, V., Metaxas, D., Sussman, M.: Textured liquids based on the marker level set. Comput. Graph. Forum 26(3), 457-466 (2007)

11. Plank, G., Burton, R., Hales, P., Bishop, M., Mansoori, T., Burton, M., Garny, A., Prassl, A., Bollensdorff, C., Mason, F., Mahmood, F., Rodriguez, B., Grau, V., Schneider, J., Gavaghan, D., Kohl, P.: Generation of histo-anatomically representative models of the individual heart: tools and application. In: Phil. Trans. Roy. Soc. (2009)

12. Sussman, M.: A parallelized, adaptive algorithm for multiphase flows in general geometries. Comput. Struct. 83(6-7), 435-444 (2005)

13. Yokoi, K., Xiao, F., Liu, H., Fukasaku, K.: Three-dimensional numerical simulation of flows with complex geometries in a regular cartesian grid and its application to blood flow in cerebral artery with multiple aneurysms. Journal of Computational Physics 202(1), 1-19 (2005) 